\title{
A simple and sensitive method to measure timing accuracy
}

\author{
ARMAND DE CLERCQ, GEERT CROMBEZ, ANN BUYSSE, and HERBERT ROEYERS \\ Ghent University, Ghent, Belgium
}

\begin{abstract}
Timing accuracy in presenting experimental stimuli (visual information on a PC or on a TV) and responding (keyboard presses and mouse signals) is of importance in several experimental paradigms. In this article, a simple system for measuring timing accuracy is described. The system uses two PCs (at least Pentium II, $200 \mathrm{MHz}$ ), a photocell, and an amplifier. No additional boards and timing hardware are needed. The first PC, a SlavePC, monitors the keyboard presses or mouse signals from the PC under test and uses a photocell that is placed in front of the screen to detect the appearance of visual stimuli on the display. The software consists of a small program running on the SlavePC. The SlavePC is connected through a serial line with a second PC. This MasterPC controls the SlavePC through an ActiveX control, which is used in a Visual Basic program. The accuracy of our system was investigated by using a similar setup of a SlavePC and a MasterPC to generate pulses and by using a pulse generator card. These tests revealed that our system has a $0.01-m s e c$ accuracy. As an illustration, the reaction time accuracy of INQUISIT for a few applications was tested using our system. It was found that in those applications that we investigated, INQUISIT measures reaction times from keyboard presses with millisecond accuracy.
\end{abstract}

Timing accuracy in both the presentation of experimental stimuli (visual information on a PC or on a TV) and responding (keyboard presses and mouse signals) is of importance for researchers using reaction time paradigms on PCs (Myors, 1998). The millisecond accuracy of older PC-DOS-based software has generally been considered as sufficient. Several methods have been described to obtain millisecond accuracy under PC DOS (see De Clercq \& Buysse, 1999; Myors, 1998). Since Microsoft Windows 95/98/ME/NT/2000 are not real-time operating systems, it is still unclear whether reaction time programs under these operating systems have millisecond accuracy. Myors (1998, 1999) has drawn attention to this problem, since experimental programs are often based on multimedia techniques.

The main aim of this article is to describe a simple and sensitive method to measure timing accuracy. Our system uses two PCs (at least Pentium II, $200 \mathrm{MHz}$ ), a necessary photocell, and an amplifier. No additionalboards and timing hardware are needed. The first PC, a SlavePC, monitors the keyboard presses or mouse signals from the PC under test and uses a photocell that is placed in front of the screen to detect the appearance of visual stimuli on the display. This SlavePC is connected through a serial line with a second PC, the MasterPC, which controls the SlavePC.

The authors thank Richard Plant, Jeff Hamm, and an anonymous reviewer for their valuable comments on a previous draft of this paper. We also thank Kemo Elektronik for allowing the publication of the diagram from the light barrier. Correspondence concerning this article should be addressed to A. De Clercq, Department of Applied Mathematics and Computer Science, Ghent University, Krijgslaan 281 S9, B9000 Ghent, Belgium (e-mail: armand.declercq@ rug.ac.be)
This setup can be used to measure the timing accuracy of the presentation of visual stimuli on a computer screen or on a TV and to measure the reaction time accuracy of keyboard presses, mouse signals, or input signals from other response consoles. Second, the accuracy of our system was tested by investigating whether generated pulses of short duration would be missed. Finally, the applicability of our system was illustrated by testing the reaction time accuracy of keyboard presses in INQUISIT from Millisecond Software, which is a software program that can be used to design experiments, such as implicit attitude tests, affective priming experiments, subliminal priming experiments, and attentional bias procedures.

\section{METHOD}

\section{General Information}

Two standard PCs are necessary to test the timing accuracy of the software of a third $\mathrm{PC}$ under test (see Figure 1). A first PC, at least a Pentium II $200 \mathrm{MHz}$, functions as a SlavePC. It runs a small program under MS-DOS and directly controls the printer and the serial port. This program detects signal changes from the printer port with a timing accuracy of up to $0.01 \mathrm{msec}$. A second PC, called the MasterPC, controls the SlavePC through a serial communication line. This MasterPC start/stops the SlavePC, defines the printer port to be used, and stores data that are captured by the SlavePC.

The SlavePC monitors when a key is pressed on the keyboard and when visual information is displayed on the screen. The keyboard signals from the PC under test are transmitted to the printer port of the Slave PC. The wiring 


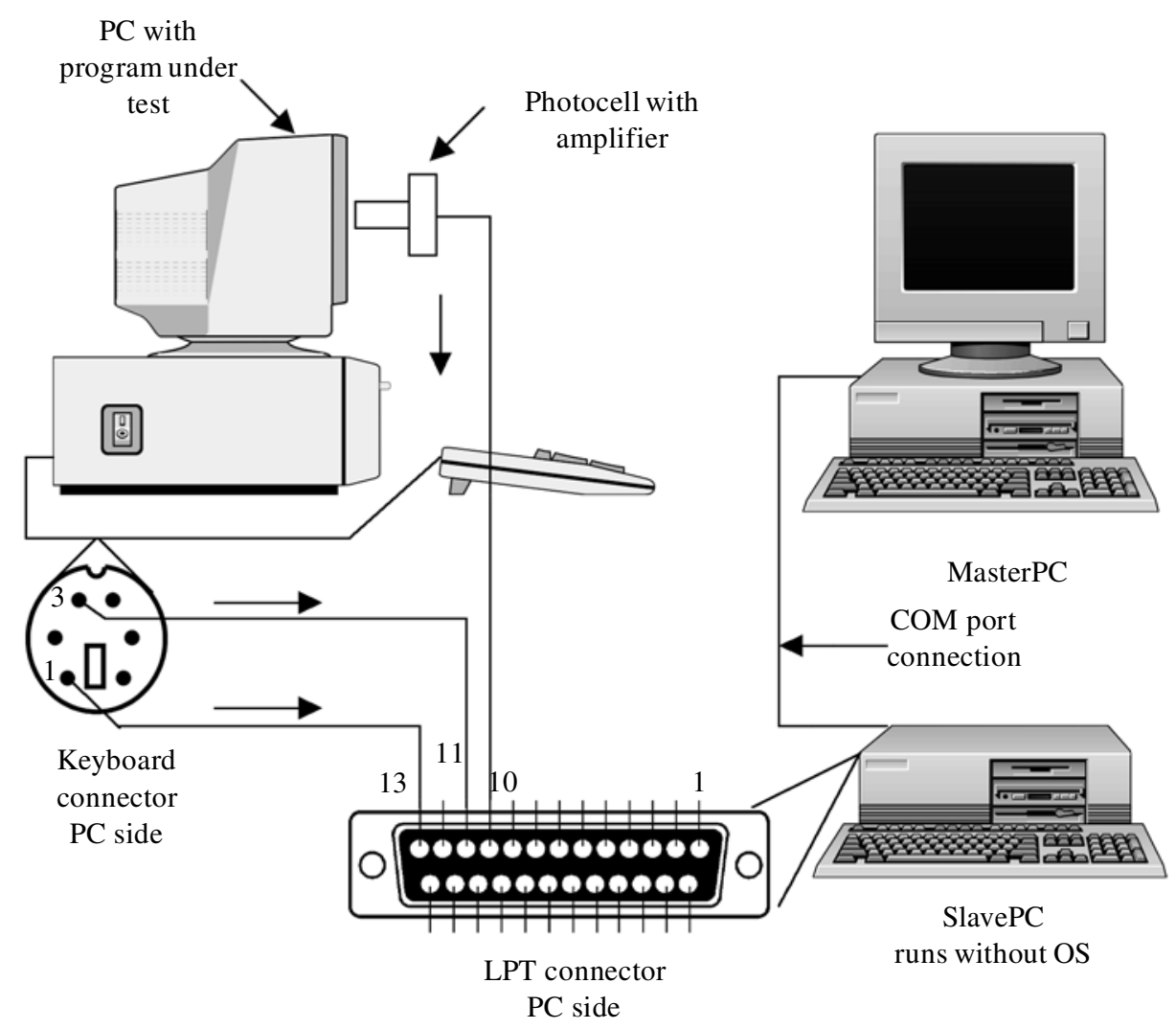

Figure 1. Experimental setup showing the wiring diagram between the keyboard of the PC under test and the photocell with the printer port of the SlavePC .

diagram is also shown in Figure 1. For measuring the timing accuracy of the software, a photocell is placed in front of the screen of the PC under test. The photocell is also connected to the printer port of the SlavePC. The photocell and the amplifier are inexpensive and available as a kit. The wiring diagram of the amplifier is shown in Figure 2 . The use of the photocell is highly accurate and flexible but requires a stimulus protocol. For the assessment of the timing accuracy of visual stimuli on a PC screen, a visual stimulus has to consist of a white square. When no visual stimulus is presented, the square is black or the whole background is black. For the assessment of the display accuracy of the video apparatus, the occurrence of missing frames is counted. Black squares have to be inserted into the video frames, and every 25 frames the black square is replaced by a white square. In both stimulus protocols, the photocellis placed where the white square will appear on the display screen.

\section{SlavePC}

At the core of the SlavePC software is a timing routine, which is activated every $10 \mathrm{msec}$. At every timer interrupt, the timing routine checks whether the signal from the parallel printer port has been changed. With standard MSDOS PC software, a high accuracy in reaction times is impossible (Graves \& Bradley, 1987). To increase the frequency of timer interrupts, several authors (Crosbie,
1989; Heathcote, 1988) have proposed modifying the timer chip (Intel 8253) by changing the value of counter 0 of the timer chip to 4A9 (hex). This results in an interrupt frequency of $1000.1522 \mathrm{~Hz}$. (Bührer, Sparrer, \& Weitku-

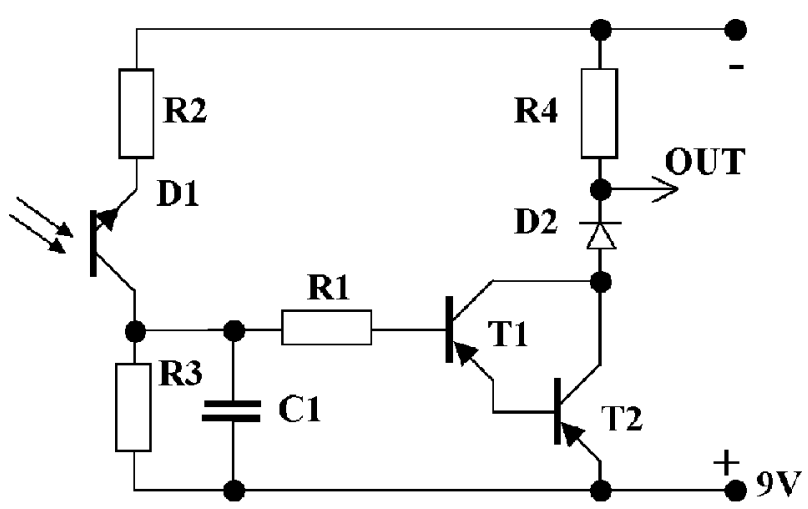

Figure 2. Parts list and wiring diagram of the photodetector. 
nat, 1987). Although faster timing routines have been described, they were until recently difficult to use in PCs (see Emerson, 1988). The Pentium 200-MHz processor, however, can easily handle high interrupt rates. In our program, the value of counter 0 was changed to 11 , resulting in an interrupt frequency of $98.610 \mathrm{KHz}$ or, otherwise stated, an interrupt every $10.14 \mathrm{msec}$. Once the program starts running, it takes complete control over the timer interrupt. In this way, possible interference with the BIOS is impossible. Timing errors such as those discussed by Bovens and Brysbaert (1990) are not possible, since the BIOS cannot update the time-of-day clock anymore. Experiments with a pulse generator card revealed that our program does not contain timing errors (see below).

The output of the photocell amplifier is connected to an input pin of the parallel port of the SlavePC. The photocell was placed before a standard computer screen (Philips 107S).The output was monitored on an oscilloscope. The rise time of the photocell is $10 \mathrm{msec}$, and the fall time is $200 \mathrm{msec}$. The rise and fall times include the values for the photocell with amplifier and the persistence of the screen phosphor. The rise time of the photocell is sufficient to detect the appearance of visual information with a millisecond accuracy, and the fall time prevents individual scan lines of the video screen from being detected as separate status changes. The photocell is attached to a metal stand with a heavy foot, which prevents accidental movements of the photocell. The timing accuracy of the SlavePC is also sufficient for detecting keypresses from a keyboard. An AT keyboard transmits data at a baud rate of approximately 10-20 KBaud, which is five times slower than the maximum frequency of the SlavePC $(98 \mathrm{KHz})$. The data from the printer port are buffered in RAM memory of the SlavePC and then transferred to the MasterPC through the serial communication line. For repetitive changes, such as a series of display frames, the software can be pro- grammed so that only the first change of a series is recorded.

The program for the SlavePC is written as generally as possible and can be started from a diskette. The port address and the baud rate for the serial port that is used for communication with the MasterPC are given as extra command line arguments at startup of the SlavePC program.

\section{MasterPC}

The software for the MasterPC is built as a set of modules (see Figure 3) and is written in Visual Basic 6. The communication with the SlavePC is controlled by an ActiveX control program, which is called RUG_SLAVE. The data from the SlavePC are logged unmodified in a text file by the program RUGFASTLOG. A separate program, RUGFASTDEC, decodes the data stored by RUGFASTLOG in a format that is compatible with programs for statistical analysis (e.g., Excel ). ${ }^{1}$

$R U G \_S L A V E$ is a modular program, which handles the communication protocol with the SlavePC and converts the data from the SlavePC to a (real) value in milliseconds. The objective is that any programmer who is familiar with Visual Basic is able to use the ActiveX module for his/her specific requirements. RUG_SLAVE uses the Microsoft MSComm control for communication with the SlavePC. An advantage is that MSComm offers control over all properties of the serial COM port without the need to program directly the hardware COM ports. The methods and properties of RUG_SLAVE are described below.

SelectComPort (PortNumber, Baudrate) initializes MSComm and selects the serial COM port and the baud rate. A baud rate of 38,400 is normally used.

Clock (property) changes the value of counter 0 of the timer chip. Four options are possible: MilliSec, HundredMicroSec, TenMicroSec, and OneTick. In the application reported below, the option TenMicroSec was selected.
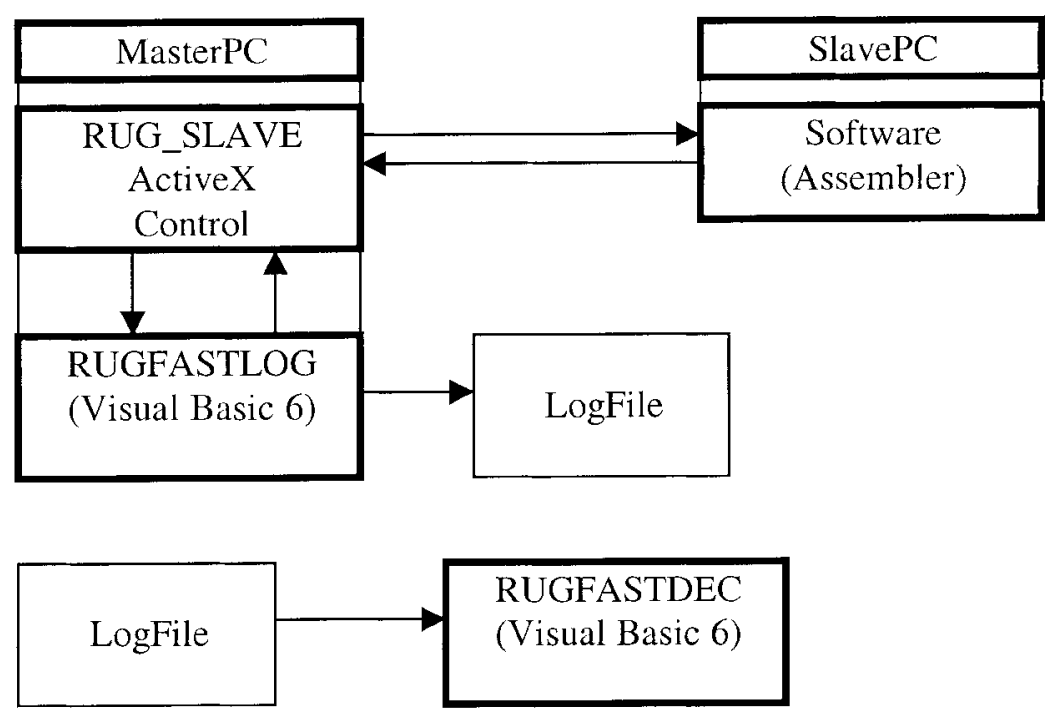

Figure 3. The software for the MasterPC is built as a set of modules. 
OUTP(Portaddress, value) and INP(Portaddress, ReturnValue) are methods with which data can be sent to and read from hardware registers in the SlavePC. These options are used during initialization of the hardware.

SlaveEnable enables (true) or disables (false) the SlavePC. SetTimOut sets the maximum time after which the SlavePC is considered as "dead."

AddChangedSlot(SlotNumber, PortAddress, Datamask, MinimalDetectTime) adds a new slot. Slots are defined to control status changes of the input pins from the LPT port of the SlavePC. These are used to detect changes from the photocell, to detect keyboard presses, and to detect mouse clicks. After this method is called for, the SlotNumber contains the number of the slots, which is used in GetData to identify the data. With Portaddress and Datamask, the address of the hardware register and the datamask are selected. MinimalDetectTime is used only for detecting changes from the photocell and allows the suppression of individual refresh frames of the computer screen. Also possible is AddFlaggedSlot for defining a serial port for data input.

GetData (SlotNumber, DataByte, DataTime) detects and reads data in a closed loop. When new data are available, SlotNumber contains the number of the slot as defined by AddChangedSlot. DataByte returns the actual data read from the parallel port, and DataTime indicates the time of the data change in a double real-number format. The value is in milliseconds.

RUGFASTLOG saves data that are transmitted from the keyboard and photocell to the parallel port of the SlavePC. It is also possible to define keyboard keys as response keys for reaction time measurement. This information is saved in the registry and will be used for selecting those keys for calculating reaction times in RUGFASTDEC. The following example shows one line of the data produced by RUGFASTLOG:

LGP 030.358

LGK 1630.404.

LGP (LoGPhotocell) indicates that this line contains data from the photocell. LGK (LoGKeyboard) indicates data input from the keyboard. The second word is the actual data from the printer port. The last word is the transition time in milliseconds. Timing data are stored in the SlavePC as long integers (32 bit), with a maximum of 4,000,000,000 counts. At every timer interrupt, the timer is incremented by 1 . Consequently, at a repetition rate of $100 \mathrm{KHz}$, the counter will reach its maximum after $11 \mathrm{~h}$. An option in RUGFASTLOG, however, allows one to produce several data files when the SlavePC is reset before each start of a new file.

RUGFASTDEC reads the data generated by RUGFASTLOG and converts it to a format compatible with programs for statistical analysis. RUGFASTLOG has different modes of operation. Only the mode for testing reaction time software is discussed here. In this mode, the program scans the input lines for the start of a series of white squares in the display frames. Next, the data lines are scanned for the start of a keyboard press. The follow- ing lines show two results in which the time is given between the appearance of the first white square frame and the time of the first signal transition of the keyboard data: RTT IS $1817.05 \mathrm{key}=\mathrm{pR}$

RTT IS $331.95 \mathrm{key}=\mathrm{pR}$.

The first two words are an abbreviation of "reaction time is." The third word is the reaction time in milliseconds. The last word refers to the key being pressed ("p").

\section{TESTING FASTLOG}

The accuracy of our system, FASTLOG, was investigated with two tests: (1) by using a similar setup of a SlavePC and MasterPC to generate pulses, and (2) by using a pulse generator card. In the first test, pulses were generated by a second setup of MasterPC and SlavePC. A specific program, called RUG_PULS, was developed and generated pulses on the output pins of the printer port of the SlavePC. The number of pulses per cycle, the duration of the pulses, and the cycle length were easily defined. The output of the printer port was then connected to the input lines from the SlavePC that was part of the setup, to assess the timing accuracy.

The first test was designed to check for missing interrupts and to check the stability of the timer chip. FASTLOG was tested for 57,000 cycles of 9 msec. Results indicated no missing data. Furthermore, the maximum deviation of the pulses was $20 \mathrm{msec}$. In the second test, pulses were generated by a PC equipped with a DAC 6602 pulse generator card from National Instruments and programmed with LabView. A series of 50-msec, 1-msec, and $10-$ msec pulses were produced. There were no missing data. The maximum deviation was $10 \mathrm{msec}$.

In a further test, the stability of the photocell was investigated with a continuous series of video frames. No frames were missed. In contrast to the previous tests, the maximum deviation was much larger-that is, $100 \mathrm{msec}$. Testing the system with an ordinary television screen showed the same result. It is as yet unclear whether the large increase in deviation occurred owing to our apparatus or owing to instability of the display refresh rate of the video screen. However, the error remains small, and the system is still adequate for assessing millisecond accuracy.

In a first application test, the FASTLOG system was used to assess the reaction time accuracy of INQUISIT. In one experiment, a word was projected onto the middle of the screen. The program waited until the user pressed one of two predefined keys and reported the reaction time. After the keypress, the word disappeared, and another word was projected. In another experiment, the words were replaced with image targets. A listing of the INQUISIT program is included in the Appendix. The results in these reaction time accuracy tests were based on the differences (in milliseconds) between the reaction times of INQUISIT and the reaction times of FASTLOG. The time that was measured with the photocell was composed of two components: the time when the image started and the time between this moment and the moment the beam reached the 
Table 1

Reaction Time Accuracy (Standard Deviation and Maximum Deviation) of Several INQUISIT Programs Using Key Board Presses

\begin{tabular}{|c|c|c|c|c|c|}
\hline Test & $\mathrm{PC}+\mathrm{OS}$ & $\begin{array}{c}\text { Standard } \\
\text { Deviation }\end{array}$ & $\begin{array}{l}\text { Maximum } \\
\text { Deviation }\end{array}$ & $\begin{array}{c}\text { Reaction Time } \\
\text { Range }\end{array}$ & $\begin{array}{c}\text { Mean } \\
\text { Reaction Times }\end{array}$ \\
\hline \multirow[t]{2}{*}{1,000 words in the center of screen } & Pentium PRO 200, Windows NT & 0.3 & \pm 0.65 & $65-1,766$ & 272 \\
\hline & Pentium II 200, Windows 98 & 0.3 & \pm 1.00 & $32-4,803$ & 282 \\
\hline 1,000 images, $640 * 480$ pixels, 24 -bit RGB color & Pentium II 200, Windows 98 & 0.34 & \pm 1.35 & $37-1,222$ & 272 \\
\hline IAT test (140 stimuli) & Pentium II 200, Windows 98 & 0.29 & \pm 0.60 & $374-1,988$ & 783 \\
\hline Sublim test & Pentium II 200, Windows 98 & 0.4 & \pm 0.80 & $150-1,823$ & 537 \\
\hline
\end{tabular}

Note-Standard deviation, maximum deviation, reaction time range, and mean reaction time are measured in milliseconds.

place on the screen where the photocell was attached. This time was a constant for all measurements. For this reason, it was subtracted from the results. For the two tests investigating reaction time accuracy to word targets, the photocell was placed in front of a white space immediately after the actual word. For the second test, white squares were used as images. For the tests investigating reaction time accuracy to colored image targets, the photocell was placed in front of the center of the image, at which a small white square was inserted. We also tested two demonstration programs of INQUISIT, named IAT and Sublim. For the IAT program, we replaced the color background with a black background and added a short trial prepause of $20 \mathrm{msec}$. For the Sublim program, we replaced the mask words with one-letter words. All images had a white square, and all mask images had a black square. These modifications were necessary to detect stimulus changes with the photocell. The first author (A.D.) was the participant in these tests. Keypresses were a, p, and 5. He explicitly varied the speed of his reaction times in order to avoid investigating reaction time accuracy only within a small range. The results of these reaction time experiments are summarized in Table 1. Table 1 also summarizes the mean reaction time of the participant and the range of reaction times. Only on some occasions was there a deviation of about $5 \mathrm{msec}$. This was the case only for the reaction time to the first stimulus presentation, when INQUISIT was used immediately after starting up the computer. Since most sessions in INQUISIT start with a training session, this is of little importance. It was found that in those examples shown, INQUISIT has a millisecond accuracy in measuring reaction times.

In a second application, we created a small assembler program that changed the printerport signals, synchonized with the video retrace signal. The signal on the printer port was measured with our procedure. The program was run in a DOS box under Windows95 and in "command prompt only" mode. This is the same procedure as that described by Myors (1999). The results of these experiments are summarized in Table 2. In comparison with Myors (1999), our method reported a much smaller value for the standard deviation while the program was run in the DOS box. The maximum deviation, however, was in the range reported by Myors. In the command prompt mode, our results were comparable to those reported by Myors (1999). The difference in accuracy between our experiment and those from Myors (1999) was probably due to the difference in method. However, the case remains that the use of a DOS box for running software for millisecond accuracy under Windows should be avoided.

In a final application to further illustrate the usefulness of our setup, we provided an example of how FASTLOG could be used to check for missing frames during playback of multimedia files on a standard computer. In reaction time experiments using multimedia files with images or sound stimuli, it is of crucial importance to have no missing frames. Missing frames are caused by a lack of processing power of the computer. Normally, the software tries to keep synchronizing the video playback by dropping frames. With a video editing program, a white square is placed in every 25 th frame. A black square is placed in all the other frames. A missing frame can be detected by measuring the time difference between the signals generated by the photocell placed in front of the computer display at the place where the squares occur.

\section{DISCUSSION}

The timing accuracy of reaction time programs has always been a major concern in research. This is especially true for software running under a multitasking operating

Table 2

Timing Accuracy of Video Retrace Programs Running Under Windows 95 (Myors Experiment)

\begin{tabular}{lllc}
\hline \multicolumn{1}{c}{ Test } & Mean & Standard Deviation & Maximum Deviation \\
\hline Video retrace full screen & & & \\
Always suspend checked, idle sensitivity low & 428.5 & 2.5 & 14.4 \\
Always suspend not checked, idle sensitivity high & 428.5 & 2.5 & 14.4 \\
Always suspend checked, idle sensitivity high & 428.4 & 2.3 & 14.4 \\
Always suspend not checked, idle sensitivity low & 428.5 & 2.4 & 14.4 \\
"Command prompt only" & 428.0 & 0.015 & 0.1 \\
\hline
\end{tabular}

Note-Mean, standard deviation, and maximum deviation are measured in milliseconds. 
system such as Windows. Although several software programs, such as E-Prime (Schneider, 1998), INQUISIT from Millisecond Software, and DMASTR (Forster \& Forster, 1999), have run reaction time experiments under Windows and all have claimed millisecond accuracy, there is a general lack of data supporting this claim. Myors (1998, 1999) was one of the first to address this important issue. He developed software that ran on the computer under test and developed special graphic techniques to test the accuracy of keypresses. Our system has the same objective. However, our method is independent from the hardware and software of the computer under test. Furthermore, only an inexpensive photocell is required, and no special hardware and no expensive software are required. Our system requires two extra computers, an easy-to-construct cable for connecting a keyboard to the printer port, and a photocell for detecting the start of images on screen. Accurate timing is guaranteed by using a fast PC DOS program on a SlavePC. The software is controlled by Windows software running on the second MasterPC and uses an ActiveX control that can be used in a Visual Basic program.

The accuracy of our system, FASTLOG, was investigated by using a similar setup of a SlavePC and MasterPC to generate pulses and by using a pulse generator card. The results indicated that our system did not miss any pulse signals. The results of both tests indicated that our setup $\log$ s with a $0.01-\mathrm{msec}$ accuracy.

As an illustration, our system was used to investigate the reaction time accuracy of INQUISIT. Responses were made with keyboard presses. Several variations of the experiment were done. In all the programs tested, INQUISIT had a millisecond accuracy in measuring reaction times. The maximum deviation between the reaction time from INQUISIT and the reaction time from our setup was always smaller than $1 \mathrm{msec}$, except in the program using picture targets. Maximum deviation was then $1.4 \mathrm{msec}$, which can still be considered as small.

All of the above-described software is free and can be downloaded from http://allserv.rug.ac.be/ adeclerc/fastlog.
The FASTLOG source code can be obtained from armand. declercq@rug.ac.be for free. The photocell and amplifier is sold by Kemo Elektronik as Light Barrier (part number, B194; cost, \$4 ). More information is available at http://www.kemo-electronic.de.

\section{REFERENCES}

Bovens, N., \& Bry SBAERT, M. (1990). IBM PC/XT/AT and PS/2 Turbo Pascal timing with extended resolution. Behavior Research Methods, Instruments, \& Computers, 22, 332- 334.

Bührer, M., Sparrer, B., \& Weit Kunat, R. (1987). Interval timing routines for the IBM PC/XT/AT microcomputer family. Behavior Research Methods, Instruments, \& Computers, 19, 327-334.

Crosbie, J. (1989). A simple Turbo Pascal 4.0 program for millisecond timing on the IBM PC/XT/AT. Behavior Research Methods, Instruments, \& Computers, 21, 408-413.

De Clerce, A., \& Buysse, A. (1999). An inexpensive system for measuring the processing of relational information by romantic partners. Behavior Research Methods, Instruments, \& Computers, 31, 299-304.

Emerson, P. L. (1988). Using serial interfaces and the C language for real-time experiments. Behavior Research Methods, Instruments, \& Computers, 20, 330-336.

Forster, K.I., \& Forster, J.C. (1999). DMASTR Home Page [On-line] Available http://www.u.arizona.edu/ kforster/dmastr/dmastr.htm.

Graves, R, \& Bradley, R. (1987). Millisecond interval timer and auditory reaction time programs for the IBM PC. Behavior Research Methods, Instruments, \& Computers, 19, 30-35.

HeAt hCote, A. (1988). Screen control and timing routines for the IBM microcomputer family using a high-level language. Behavior Research Methods, Instruments, \& Computers, 20, 289-297.

Myors, B. (1998). A simple graphical technique for assessing timer accuracy of computer systems. Behavior Research Methods, Instruments, \& Computers, 30, 454-456.

Myors, B. (1999). Timing accuracy of PC programs running under DOS and Windows. Behavior Research Methods, Instruments, \& Computers, 31, 322-328.

SCHNEIDER, W. (1998, November). E-Prime: A cross platform experiment generator studio for computerized behavioral research. Paper presented at the Commercial Symposium of the 28th Annual Conference of the Society for Computers in Psychology, Dallas.

\section{NOTE}

1. To avoid name conflicts with other registered software, the prefix RUG_is added to all our controls. 
APPENDIX

Listing of the Inquisit Code Used in the Word Test

$<$ defaults $>$

$/$ screencolor $=(0,0,0)$

/ font = ("Courier", -18, 700, 0, 49)

/ txbgcolor $=(255,255,255)$

$/$ pretrialpause $=1750$

$<$ /defaults $>$

$<$ text standaardpos $>$

$/$ numitems $=1$

/ items = ("WORD ")

$/$ select $=$ random

$/$ erase $=\operatorname{true}(0,0,0)$

$</$ text $>$

$<$ text pleasantreminder $>$

/ numitems $=1$

$/$ items $=($ "p")

$/$ position $=(85,8) \mathrm{p}$

$</$ text $>$

$<$ text unpleasantreminder

/ numitems = 1

$/$ items $=$ ("a")

$/$ position $=(15,8)$

$</$ text $>$

$<$ instruct $>$

/ nextkey $=($ "p")

/ prevkey $=$ ("a")

$/$ font = ("Arial", -20, 400, 0, 34)

$<$ instruct $>$

$<$ page StartUp $>$

GO
$</$ page $>$

$<$ page end $>$

$\mathrm{OK}$

$<$ page $>$

$<$ trial TRIAL1>

/ validresponse $=($ "a", "p")

/ correctresponse $=$ ("p")

$/$ frames $=$ [1=standaardpos $]$

$</$ trial $>$

$<$ block BLOCK1>

$/$ bgstim $=($ pleasantreminder, unpleasantreminder $)$ trials $=[1-5=$ TRIAL1 $]$

/ blockfeedback = (latency, correct $)$

$<$ block $>$

$<$ expt $>$

$/$ preinstructions $=(\operatorname{Start} U p)$

$/$ blocks $=[1=$ BLOCK 1$]$

$/$ postinstructions $=($ end $)$

$</$ expt $>$

The experiment with images is essentially the same program, where the $<$ text standaardpos $>$ block is replaced by the following: $<$ picture standaardpos $>$

$/$ numitems $=5$

$/$ items $=$

(“B1.bmp",'B2.bmp",,'B3.bmp",,'B4.bmp",,'B5.bmp")

$/$ select $=$ random

$/$ erase $=\operatorname{true}(0,0,0)$

$<$ picture $>$

(Manuscript received December 27, 2001;

revision accepted for publication July 8, 2002.) 